\title{
Sexual differences in exploration behavior in Xenopus tropicalis?
}

\author{
Mathieu Videlier ${ }^{1}$, Raphaël Cornette ${ }^{2}$, Camille Bonneaud ${ }^{3}$ and Anthony Herrel ${ }^{1,4, *}$
}

\begin{abstract}
The two sexes of a species often differ in many ways. How sexes differ depends on the selective context, with females often investing more in reproductive output and males in territory defense and resource acquisition. This also implies that behavioral strategies may differ between the two sexes, allowing them to optimize their fitness in a given ecological context. Here, we investigated whether males and females differ in their exploration behavior in an aquatic frog (Xenopus tropicalis). Moreover, we explored whether females show different behavioral strategies in the exploration of a novel environment as has been demonstrated previously for males of the same species. Our results show significant sex differences, with males exploring their environment more than females. Yet, similar to males, female exploratory behavior varied significantly among individuals and broadly fell into three categories: shy, intermediate and bold. Moreover, like in males, behavioral strategies are decoupled from morphology and performance. Our results suggest that females are more sedentary than males, with males engaging in greater risk taking by exploring novel environments more. Male and female behaviors could, however, be classified into similar groups, with some individuals being bolder than others and displaying more exploration behavior. The decoupling of morphology and performance from behavior appears to be a general feature in the species and may allow selection to act on both types of traits independently.
\end{abstract}

KEY WORDS: Performance, Morphology, Frog, Locomotion, Sexual dimorphism

\section{INTRODUCTION}

Exploration behavior was defined by Scott as a sensory inspection of the environment (Scott, 1956). Exploration behavior is crucial as it allows animals to find food, reproductive partners and new territories. Moreover, longer distance dispersal is also driven to a large extent by individual exploration behavior. Different dispersal strategies have been observed in animals, with some being regular such as migration (Pulido et al., 2001) and others more episodic. The latter dispersal strategy is typically associated with escape from habitats that are being modified (Berg et al., 2010; Wittern and Berggren, 2007), and may be important to population survival in the context of resource limitation or catastrophic events. Whether an animal has a tendency to disperse is largely determined by its personality. Two contrasting behavioral syndromes are typically

${ }^{1}$ UMR 7179 CNRS/MNHN, Département d'Ecologie et de Gestion de la Biodiversité, 57 rue Cuvier, Case postale 55, Paris 75231, Cedex 5, France.

${ }^{2}$ Origine, Structure et Evolution de la Biodiversité, UMR 7205 CNRS/MNHN, 45 rue Buffon, Paris 75005, France. ${ }^{3}$ Centre for Ecology \& Conservation, College of Life and Environmental Sciences, University of Exeter, Penryn, Cornwall TR10 9FE, UK. ${ }^{4}$ Evolutionary Morphology of Vertebrates, Department of Biology, Ghent University, K. L. Ledeganckstraat 35, Gent B-9000, Belgium.

*Author for correspondence (anthony.herrel@mnhn.fr)

Received 5 February 2015; Accepted 9 April 2015 identified in this context: bold and shy (Sih et al., 2004). These two syndromes are typically fixed throughout the life of an individual and have been demonstrated in a wide variety of animals including invertebrates such as crabs (Decker and Griffen, 2012) and crickets (Niemelä et al., 2012), but also in many vertebrates including birds (Carere et al., 2005), fish (Dzieweczynski and Crovo, 2011; Brown et al., 2007), turtles (Mafli et al., 2011) and primates (Uher et al., 2008). Bold individuals show a tendency to explore their environment more, thus increasing the risk of predation but also increasing the probability of finding mates, food or new territories. Shy individuals typically explore less, thus decreasing risk taking but also reducing opportunities. On average, these two strategies show equal fitness in neutral conditions, resulting in the maintenance of both (Smith and Blumstein, 2008).

Interestingly, not all individuals behave similarly and several factors are known to affect an animal's behavior including age and sex (Carere et al., 2005; Dingemanse and Réale, 2005). Differences between the sexes may arise as a result of sexual selection (Hedrick and Temeles, 1989) and result in differences in body size (Woolbright, 1983) and other morphological traits (Desjardins and Fernald, 2009). As the energetic cost of gamete production strongly differs between the sexes, this often results in differences in body size and life-history traits (Monroe and Alonzo, 2014). Moreover, exploration behavior may also be different in males and females because of the association between male exploration and territorial aggressiveness, as has been shown in birds and fish (Carere et al., 2005; Brown et al., 2007; Dzieweczynski and Crovo, 2011). In some birds, females also prefer bolder males and consequently sexual selection may also impact the evolution of exploration behavior (Dingemanse and Réale, 2005). Moreover, hormonal differences between the sexes are likely to impact their exploration behavior (Brandner, 2007). For example, in humans, men explore over greater distances than women do. This is caused by differences in orientation ability as well as constraints due to parental care that are not the same in the two sexes (Brandner, 2007).

Amphibians, and especially frogs, are of interest in this context for two reasons: (1) the majority of species are dimorphic, with females typically being larger than males (Monnet and Cherry, 2002) and (2) amphibians are characterized by a low overall mobility, making them especially vulnerable to local habitat destruction and habitat fragmentation (Hillers et al., 2008). Thus, dispersal is likely under strong selection in fragmented habitats. Given known sexual dimorphism in morphology in many amphibian species (Monnet and Cherry, 2002; Shine, 1979), the two sexes may not be impacted to the same degree, however. Surprisingly, amphibian exploratory behavior remains poorly investigated. Here, we focused on Xenopus (Silurana) tropicalis (Gray 1864) as a model because this species is sexually dimorphic in body size, limb dimensions and locomotor performance (Herrel et al., 2012). The natural habitat of this species (the coastal rain forest belt of West Africa) is increasingly being fragmented as a result of direct human impacts and climate change (Hillers et al., 2008). This likely imposes selective pressures on locomotion and 
may affect the survival of animals with different exploration behaviors. An investigation of exploration behavior in male $X$. tropicalis showed a dissociation between performance and morphology on the one hand and exploration behavior on the other hand, suggesting that selection could act on behavior without affecting locomotor performance (Videlier et al., 2014). This circumvents the trade-off between endurance, important in the exploration of novel environments, and burst performance capacity, which is important in prey capture and predator escape.

We concentrated on the exploration behavior in females given the known differences in morphology and performance between the sexes. We specifically tested (1) whether females show behavioral syndromes related to the exploration of a novel environment, (2) whether behavioral strategies are similar in the two sexes and (3) whether and how the sexes differ in their overall exploration behavior. To complete, we tested whether exploration behavior is indeed decoupled from morphology and performance in females, as well as at the population level.

\section{RESULTS}

\section{Female exploration behavior}

Female $X$. tropicalis began to move $426 \mathrm{~s}$ after the onset of the experiment on average (range: 5.49-1639 s) and stopped moving $2348 \mathrm{~s}$ after the start of the trial on average (range: 145-3601 s). They covered a mean distance of $1.5 \mathrm{~m}$ in $1 \mathrm{~h}$ (range: $23-6393 \mathrm{~cm}$ ). The mean duration of a roundtrip was $206 \mathrm{~s}$ (range: 29-1357 s) when including pauses and $13 \mathrm{~s}$ (range: $2.81-44 \mathrm{~s}$ ) when excluding pauses. The pause is an important part of the exploration behavior as it allows an individual to screen its environment. Animals stopped moving 1.97 times on average (range: 0.5-12 times). Females moved with a mean overall speed of $5.36 \mathrm{~cm} \mathrm{~s}^{-1}$ (range: 0.06 $22.10 \mathrm{~cm} \mathrm{~s}^{-1}$ ) and a mean movement speed of $15.24 \mathrm{~cm} \mathrm{~s}^{-1}$ (range: 1.39-42.75 $\mathrm{cm} \mathrm{s}^{-1}$; see Table 1).

Three different groups were identified (35 individuals in the first, 21 in the second and four in the last; Fig. 1) in the Gaussian mixture model analysis using the 13 repeatable variables without imposing the number of groups. The three clusters showed differences in exploration behavior (Wilk's $\lambda=0.02, F_{26,90}=19.74, P<0.001$ ). Subsequent ANOVA showed significant differences for all variables $(P<0.05)$, except the average number of pauses, which did not differ between the three groups $\left(F_{2,57}=1.25, P=0.30\right)$. Group 1 showed numerous movements compared with the two others. Group 2 showed the opposite behavioral pattern, characterized by a reduced exploration of the tank. Group 3 showed an intermediate level of exploration as indicated by the number of movements, the distance traveled and the duration of exploration (Table 2). When testing whether the groups identified differed in morphology and performance, no significant differences were detected in body size, head, pelvic girdle or limb dimensions (see Table 3). Finally, no significant differences in locomotion performance were detected between the three groups (Wilk's $\lambda=0.82, F_{10,78}=0.79, P=0.64$ ).

\section{Sexual dimorphism in exploration behavior}

When comparing the two sexes, males were found to move more quickly (average speed: $12.25 \mathrm{~cm} \mathrm{~s}^{-1}$ for males versus $5.36 \mathrm{~cm} \mathrm{~s}^{-1}$ for females) and showed fewer pauses (average number of pauses in males: 1.31 versus 1.97 in females), resulting in a shorter average roundtrip duration (average duration: $99.07 \mathrm{~s}$ in males versus $205.86 \mathrm{~s}$ in females). Moreover, the time spent hidden showed significant differences, with males spending more time hiding than females (average time spent hidden: $299.05 \mathrm{~s}$ in males versus $150.78 \mathrm{~s}$ in females). A MANOVA performed on the behavioral
Table 1. Exploration behavior in female Xenopus tropicalis

\begin{tabular}{|c|c|c|c|}
\hline & Average & Maximal & Minimal \\
\hline Number of roundtrips & 10.04 & 40.00 & 0.03 \\
\hline Number of complete roundtrips & 7.58 & 37.33 & 0.00 \\
\hline Number of movements & 21.78 & 79.67 & 0.67 \\
\hline Total distance moved (cm) & 1496.35 & 6393.16 & 23.08 \\
\hline Average speed $\left(\mathrm{cm} \mathrm{s}^{-1}\right)$ & 5.36 & 22.10 & 0.06 \\
\hline Maximal speed $\left(\mathrm{cm} \mathrm{s}^{-1}\right)$ & 12.27 & 49.72 & 0.06 \\
\hline Minimal speed $\left(\mathrm{cm} \mathrm{s}^{-1}\right)$ & 1.67 & 18.38 & 0.03 \\
\hline Average speed without pauses $\left(\mathrm{cm} \mathrm{s}^{-1}\right)$ & 15.24 & 42.75 & 1.39 \\
\hline Maximal speed without pauses $\left(\mathrm{cm} \mathrm{s}^{-1}\right)$ & 23.00 & 112.44 & 1.39 \\
\hline Minimal speed without pauses $\left(\mathrm{cm} \mathrm{s}^{-1}\right)$ & 9.18 & 37.24 & 1.39 \\
\hline Average duration of a roundtrip (s) & 205.86 & 1356.89 & 28.76 \\
\hline Maximal duration of a roundtrip (s) & 570.02 & 2023.38 & 64.27 \\
\hline Minimal duration of a roundtrip (s) & 93.37 & 1093.96 & 0.71 \\
\hline $\begin{array}{l}\text { Average duration of a roundtrip without } \\
\text { pauses (s) }\end{array}$ & 12.64 & 44.12 & 2.81 \\
\hline $\begin{array}{l}\text { Maximal duration of a roundtrip without } \\
\text { pauses (s) }\end{array}$ & 28.71 & 121.71 & 2.96 \\
\hline $\begin{array}{l}\text { Minimal duration of a roundtrip without } \\
\text { pauses (s) }\end{array}$ & 5.84 & 28.47 & 0.71 \\
\hline Latency of the first movement (s) & 426.32 & 1639.22 & 5.49 \\
\hline Latency of the second movement (s) & 560.67 & 1752.56 & 0.00 \\
\hline Latency of the last movement (s) & 2347.91 & 3601.84 & 144.53 \\
\hline Duration of all movements with pauses (s) & 1191.86 & 3032.24 & 100.24 \\
\hline Duration of exploration without pauses (s) & 124.14 & 411.18 & 4.87 \\
\hline Total time spent hidden (s) & 878.76 & 2539.66 & 0.00 \\
\hline Average time spent hidden (s) & 150.78 & 837.92 & 0.00 \\
\hline Maximal time spent hidden (s) & 393.26 & 1224.47 & 0.00 \\
\hline Minimal time spent hidden (s) & 57.05 & 806.62 & 0.00 \\
\hline Average number of pauses & 1.97 & 10.22 & 0.50 \\
\hline Maximal number of pauses & 3.73 & 12.00 & 0.67 \\
\hline Minimal number of pauses & 0.79 & 9.00 & 0.00 \\
\hline Number of movements away from the wall & 2.54 & 8.67 & 0.00 \\
\hline
\end{tabular}

Highlighted variables were not repeatable across trials.

variables showed significant differences between the sexes (Wilk's $\left.\lambda=0.31, F_{15,80}=11.79, P<0.001\right)$. Subsequent ANOVA showed that speed (average, minimal and maximal), the duration of a roundtrip, the number of pauses and the time spent hidden showed significant differences between the sexes (Fig. 2, Table 4).

The combined data set for 37 males and 61 females included 15 behavioral variables that were found to be repeatable. Our

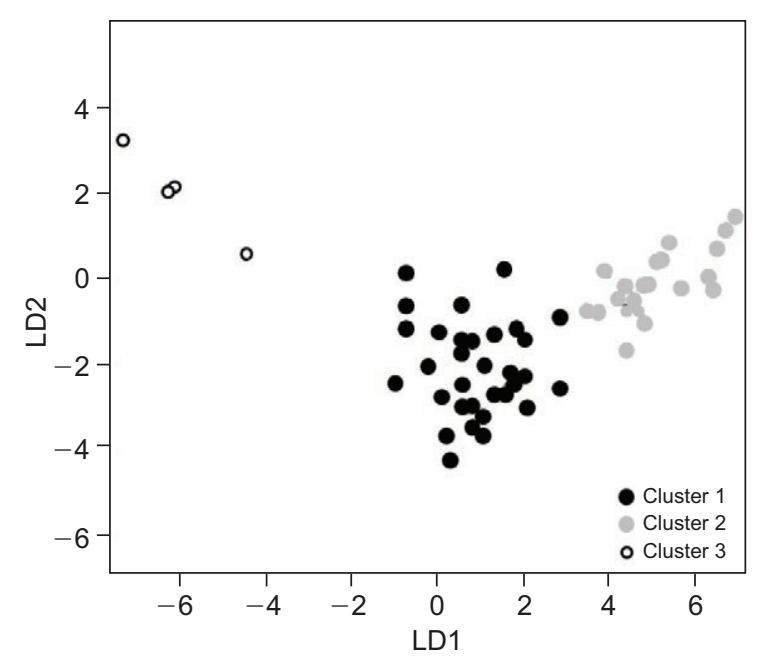

Fig. 1. Discriminate function based on the repeatable exploration behavior variables in females. The three (bold, shy and intermediary) behavioral syndromes are indicated (clusters $1-3$, respectively). 
Table 2. Results of MANOVA testing for differences between behavioral groups in female $X$. tropicalis

\begin{tabular}{lccc}
\hline & $F$ & $P$ & $\begin{array}{l}\text { Group } \\
\text { difference }\end{array}$ \\
\hline Total distance moved & 98.08 & $<0.001$ & $3<1<2$ \\
Number of roundtrips & 68.75 & $<0.001$ & $3<1<2$ \\
Number of complete roundtrips & 92.94 & $<0.001$ & $3<1<2$ \\
Number of movements & 87.28 & $<0.001$ & $3<1<2$ \\
Maximal speed & 72.73 & $<0.001$ & $3<1=2$ \\
Number of movements away from the wall & 19.10 & $<0.001$ & $3<1<2$ \\
Average number of pauses & 1.25 & 0.29 & $3=1=2$ \\
Maximal number of pauses & 6.07 & $<0.001$ & $3=1<2$ \\
Minimal number of pauses & 10.40 & $<0.001$ & $\mathbf{2 = 1 < 3}$ \\
Latency of the last movement & 9.46 & $<0.001$ & $\mathbf{3}=1<2$ \\
Minimal duration of a roundtrip without & 7.80 & $<0.001$ & $\mathbf{2 = 1 < 3}$ \\
$\quad$ pauses & & & \\
Maximal duration of a roundtrip without & 9.42 & $<0.001$ & $\mathbf{3 = 1 < 2}$ \\
$\quad$ pauses & & & \\
Duration of exploration without pauses & 43.29 & $<0.001$ & $\mathbf{3}<1<2$ \\
\hline
\end{tabular}

Gaussian mixture analysis performed on the combined data set identified three groups (56, four and 36 individuals, respectively). A MANOVA performed on the behavioral variables indicated significant differences between groups (Wilk's $\lambda=0.02$, $\left.F_{30,158}=31.90, P<0.001\right)$. ANOVA showed significant differences in all variables $(P<0.05)$ with the exception of the latency to the first movement, the maximal duration of a roundtrip and the average of number of pauses (Table 4). One group showed fewer and slower movements compared with the two others. The other two groups were generally similar, with the exception of the duration of a roundtrip, the number of pauses and the speed-related variables (Table 4). MANOVA testing for differences between the three groups showed significant differences in morphology $(P<0.05)$, but not in performance (Wilk's $\lambda=0.85, F_{10,150}=1.24, P=0.27$ ). Subsequent ANOVA and post hoc tests showed that the differences in morphology were significant when comparing groups 1 and 3 (Table 5).

\section{DISCUSSION}

\section{Female exploratory behavior}

Across all females, three distinct and robust behaviors were detected using clustering analyses without a priori group definition. Two major and opposing behavioral syndromes, bold and shy, are typically identified in many species of animals including invertebrates such as spiders (Kralj-Fišer and Schneider, 2012) and crabs (Watanabe et al., 2012), but also a variety of vertebrates including rodents (Shillito, 2013), birds (Dingemanse et al., 2006) and fish (Wilson and Godin, 2009). Bold individuals typically explore more and make fewer stops or pauses. Yet, this pattern of exploration exposes an individual to risks such as

Table 3. Results of MANOVA testing for differences in locomotor performance and limb morphology across behavioral groups for females and the entire data set combining males and females

\begin{tabular}{|c|c|c|c|c|c|c|}
\hline & \multicolumn{3}{|l|}{ Females } & \multicolumn{3}{|c|}{ Males and females } \\
\hline & Wilk's $\lambda$ & $F$ & $P$ & Wilk's $\lambda$ & $F$ & $P$ \\
\hline Performance & 0.82 & 0.79 & 0.64 & 0.85 & 1.25 & 0.27 \\
\hline Body size & 0.93 & 0.95 & 0.44 & 0.67 & 9.64 & $<0.001$ \\
\hline Head measures & 0.86 & 0.98 & 0.46 & 0.81 & 2.33 & 0.02 \\
\hline Pelvic girdle dimensions & 0.97 & 0.38 & 0.82 & 0.71 & 8.03 & $<0.001$ \\
\hline Forelimb measures & 0.75 & 1.49 & 0.15 & 0.80 & 1.99 & 0.04 \\
\hline Hindlimb measures & 0.83 & 0.95 & 0.49 & 0.73 & 2.91 & $<0.01$ \\
\hline
\end{tabular}

Bold indicates significant $P$-values. predation (Smith and Blumstein, 2008). At the opposite end of the spectrum, shy individuals explore less and spend more time hidden, but take fewer risks. However, these two strategies are likely to have similar overall fitness in the animal's natural unmodified environment. Whereas bold individuals may encounter more resources and sexual partners that allow them to increase the number of offspring, their overall survival may be decreased as a result of the greater risks taken (van Oers et al., 2004; Wolf et al., 2007). In female $X$. tropicalis the two major variables discriminating between bold and shy individuals are the speed of movement and the latency of the last movement. Bold females move fast, yet stop moving earlier.

The data sets for both females (this study) and males (Videlier et al., 2014) suggest the presence of a third pattern of exploration. This pattern has characteristics that are intermediate between the extremes of bold and shy. The individuals showing an intermediate pattern maintained some specific traits of both bold and shy individuals. Intermediate females moved faster and explored longer than shy ones during longer exploration events. However, during short exploration events they were similar to shy individuals, taking many pauses and stopping movements earlier. Both the number of pauses and the duration of a roundtrip are likely strongly linked to the overall risks encountered during exploration; while pauses allow an individual to screen the environment and thus decrease risks, short roundtrips limit the overall exposure to risks. Interestingly, females showing this intermediate strategy modulate their behavior depending on the exploratory phase, taking less risk in short bouts of exploration, yet greater risk during long ones. As movement speed is an important parameter in the context of predator escape (Husak et al., 2008), intermediate females may benefit from a faster exploration when engaging in longer exploration bouts. These intermediate individuals will likely have similar fitness to bold and shy ones because of their ability to balance risk taking and survival (van Oers et al., 2004).

\section{Sexual dimorphism in exploration behavior}

When comparing our data for females with previously published data for males of the same species (Videlier et al., 2014), we were able to identify similarities among groups of behaviors. Indeed, three distinct exploration behaviors were identified in each case: the 'classic' bold and shy groups, as well as an intermediate group. However, the intermediate group was not strictly identical in males and females. While intermediate males were characterized by a long latency to initiate the exploration of their environment and are thus more similar to shy individuals, they displayed exploratory behavior that was more similar to that of bold individuals. In females, however, intermediate animals explored at the same speed as bold individuals did, but stopped moving earlier, similar to shy ones. Moreover, the intermediate females appeared to modulate their behavior depending on the duration of exploration. Another interesting similarity between males and females was the relatively low number of shy individuals detected (four females and five males). This low number might be explained by two different scenarios. Firstly, as one of the principal characteristics of shy individuals is to reduce risk taking by staying hidden, the probability of capture is likely lower. Thus, it is relatively common to find only a few shy individuals (Carter et al., 2012; Garamszegi et al., 2009). Secondly, a population often involves the presence of many individuals with one trait and few with an alternative trait. When the environmental context changes, however, the rare trait may become advantageous and invade the entire population at the expense of the initially common trait (Hedrick, 2007). As such, it may remain fixed in the population at low frequency. 


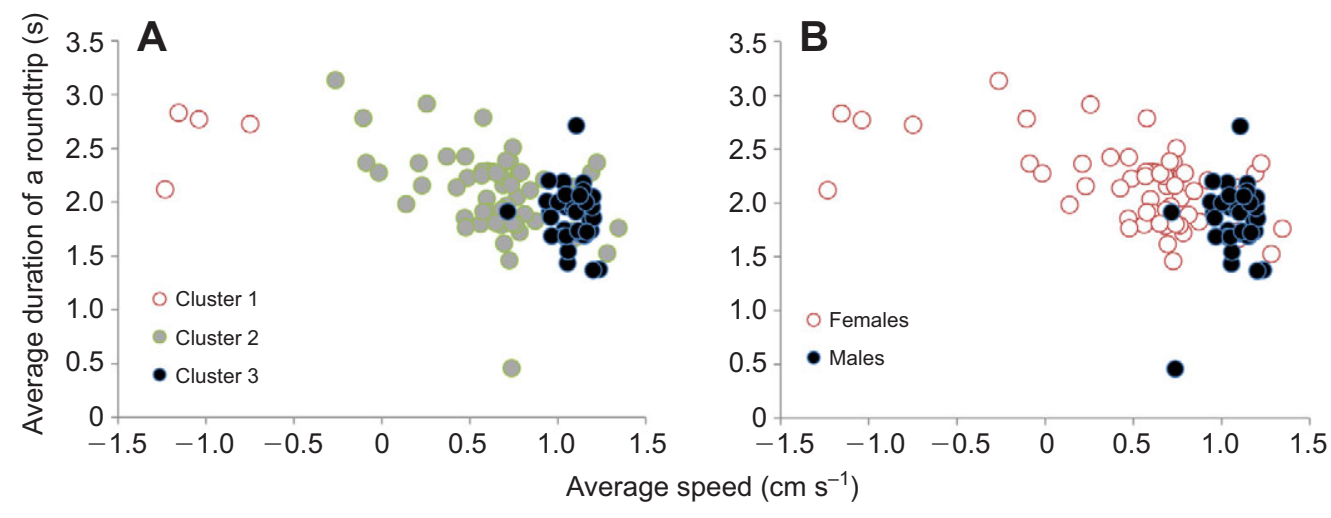

Fig. 2. Graphs of average duration of a roundtrip as a function of average speed. (A) The three behavioral groups identified with the Gaussian mixture analysis are indicated (clusters 1-3). (B) The sex of the individuals is indicated, with open symbols representing females and filled symbols representing males.
When analyzing the data for all individuals of both sexes combined, three groups could be identified as well. Among these three groups, one corresponded to shy individuals, whereas the other two appeared to correspond to bold individuals. Analyses of behavioral traits differing between groups indicated that these groups varied in speed of movement, average duration of a roundtrip and the number of pauses taken. Group 3 appeared to be the boldest and was composed entirely of males, suggesting that, on average, males were bolder than females in their exploration behavior (Fig. 2, Table 4). When comparing sex differences in exploration behavior, we found that males moved more and more quickly and took fewer pauses, resulting in shorter average roundtrip times. Dispersal in $X$. tropicalis is typically observed during the rainy season and is facilitated by both the establishment of new, but temporary, aquatic connections between ponds and overland dispersal during periods of heavy rain. Males are likely more mobile than females as male frogs in general need to find as many sexual partners as possible (Wells, 1977). Boldness is often connected to aggression (Mafli et al., 2011; Wilson and Godin, 2009) and Xenopus males are known to engage in male-male competitive fighting (Rabb and Rabb, 1965; Rabb, 1969; Wells, 1977). Moreover, females in some species of animals are known to have a preference for bolder males, which may impose selection on male behavior (Dingemanse and Réale, 2005; Godin and Dugatkin, 1996; Smith and Blumstein, 2008). The relationship between boldness and aggressive behavior is thought to be related to the levels of circulating hormones such as cortisol, testosterone and melanin, which are typically higher in males (Dingemanse and Goede, 2004; Mafli et al., 2011; Thomson et al., 2011).

\section{Decomposition of locomotion patterns: the dissociation of exploratory behavior and performance/morphology}

A previous study of male $X$. tropicalis revealed that individuals in the three behavioral groups did not significantly differ in morphology and performance (Videlier et al., 2014). The fact that a similar result was observed for females suggests that this disconnect may be a general feature of this species. However, when analyzing behavioral variation across both males and females, differences among behavioral groups in morphology, but not performance, were detected. Specifically, differences in morphology were significant between groups 1 and 3. However, an exploration of the data shows that this difference also corresponds to differences between males (group 3) and females (group 1). This result is consistent with previously reported sex differences in morphology (Herrel et al., 2012). Thus, mobility in $X$. tropicalis appears to be composed of two independent sets of traits: the exploration behavior on the one hand and performance/morphology on the other hand.

In the context of the ongoing habitat fragmentation, selection on overall mobility is likely great because of the increase in the distance between optimal habitat sites (Hillers et al., 2008). The maintenance of niche networks is, however, necessary to maintain gene flow within and between populations. Without gene flow, the

Table 4. Results of ANOVA testing for differences in exploration behavior across the behavioral groups identified in the data set consisting of data for males and females combined

\begin{tabular}{|c|c|c|c|c|c|c|}
\hline & \multicolumn{3}{|c|}{ Behavior } & \multicolumn{3}{|l|}{ Sex } \\
\hline & $F$ & $P$ & Group & $F$ & $P$ & $\begin{array}{l}\text { Group } \\
\text { difference }\end{array}$ \\
\hline Total distance & 23.18 & $<0.001$ & $2<3=1$ & 0.85 & 0.36 & Male $=$ female \\
\hline Number of complete roundtrips & 8.85 & $<0.001$ & $2<3=1$ & 0.00 & 0.97 & Male $=$ female \\
\hline Total number of movements & 18.57 & $<0.001$ & $2<3=1$ & 1.14 & 0.29 & Male $=$ female \\
\hline Number of movements away from wall & 6.78 & $<0.01$ & $2<3=1$ & 0.62 & 0.43 & Male $=$ female \\
\hline Time of all movements with pauses & 12.01 & $<0.001$ & $2<3=1$ & 0.39 & 0.53 & Male $=$ female \\
\hline Average time spent hidden & 58.07 & $<0.001$ & $2<3=1$ & 9.02 & $<0.01$ & Male>female \\
\hline Average speed & 122.60 & $<0.001$ & $2<3<1$ & 35.91 & $<0.001$ & Male>female \\
\hline Maximal speed & 138.10 & $<0.001$ & $2<3<1$ & 11.64 & $<0.001$ & Male >female \\
\hline Minimal speed & 84.66 & $<0.001$ & $2<3<1$ & 132.30 & $<0.001$ & Male>female \\
\hline Average duration of a roundtrip & 9.82 & $<0.01$ & $3<1<2$ & 18.36 & $<0.001$ & Male<female \\
\hline Maximal duration of a roundtrip & 2.33 & 0.10 & $2=1=3$ & 10.77 & $<0.01$ & Male<female \\
\hline Average number of pauses & 5.02 & 0.01 & $(1<3)=2$ & 11.92 & $<0.001$ & Male<female \\
\hline Latency of the first movement & 2.23 & 0.11 & $2=1=3$ & 0.16 & 0.69 & Male $=$ female \\
\hline Latency of the second movement & 93.10 & $<0.001$ & $2<3=1$ & 1.98 & 0.16 & Male $=$ female \\
\hline Latency of the last movement & 5.20 & $<0.01$ & $(3<2)=1$ & 3.11 & 0.08 & Male $=$ female \\
\hline
\end{tabular}

Bold values indicate significant $P$-values and highlighted cells indicate variables with significant differences between clusters 1 and 3 . 
Table 5. Results of ANOVA testing for differences in locomotor performance and limb morphology across the behavioral groups identified in the data set consisting of data for males and females combined

\begin{tabular}{|c|c|c|c|}
\hline & $F$ & $P$ & $\begin{array}{l}\text { Group } \\
\text { difference }\end{array}$ \\
\hline \multicolumn{4}{|l|}{ Body size } \\
\hline Snout-vent length (mm) & 9.19 & $<0.001$ & $(1=2) \neq 3$ \\
\hline Mass (g) & 20.11 & $<0.001$ & $(1=2) \neq 3$ \\
\hline \multicolumn{4}{|l|}{ Head dimensions } \\
\hline Length (mm) & 3.45 & 0.04 & $2=(1 \neq 3)$ \\
\hline Width (mm) & 4.27 & 0.02 & $2=(1 \neq 3)$ \\
\hline Height (mm) & 8.41 & $<0.001$ & $2=(1 \neq 3)$ \\
\hline Lower jaw length (mm) & 0.98 & 0.38 & $1=2=3$ \\
\hline \multicolumn{4}{|l|}{ Pelvic girdle } \\
\hline Ilium length (mm) & 16.16 & $<0.001$ & $2=(1 \neq 3)$ \\
\hline Ilium width (mm) & 7.50 & $<0.001$ & $(1=2) \neq 3$ \\
\hline \multicolumn{4}{|l|}{ Forelimb segments } \\
\hline Humerus (mm) & 0.90 & 0.41 & $1=2=3$ \\
\hline Radius (mm) & 1.07 & 0.35 & $1=2=3$ \\
\hline Hand $(\mathrm{mm})$ & 2.33 & 0.10 & $1=2=3$ \\
\hline Finger (mm) & 0.24 & 0.78 & $1=2=3$ \\
\hline Forelimb length (mm) & 0.38 & 0.69 & $1=2=3$ \\
\hline \multicolumn{4}{|l|}{ Hindlimb segments } \\
\hline Femur (mm) & 2.71 & 0.07 & $1=2=3$ \\
\hline Tibia (mm) & 10.92 & $<0.001$ & $2=(1 \neq 3)$ \\
\hline Foot (mm) & 9.30 & $<0.001$ & $2=(1 \neq 3)$ \\
\hline Toe $(\mathrm{mm})$ & 1.76 & 0.18 & $1=2=3$ \\
\hline Hindlimb length (mm) & 1.36 & 0.26 & $1=2=3$ \\
\hline \multicolumn{4}{|l|}{ Performance } \\
\hline Average swimming speed $\left(\mathrm{cm} \mathrm{s}^{-1}\right)$ & 0.43 & 0.65 & $1=2=3$ \\
\hline Peak swimming speed $\left(\mathrm{cm} \mathrm{s}^{-1}\right)$ & 0.04 & 0.96 & $1=2=3$ \\
\hline Peak swimming acceleration $\left(\mathrm{cm} \mathrm{s}^{-2}\right)$ & 2.45 & 0.09 & $1=2=3$ \\
\hline Maximal time jumped (s) & 1.91 & 0.15 & $1=2=3$ \\
\hline Maximal distance jumped (cm) & 0.25 & 0.78 & $1=2=3$ \\
\hline
\end{tabular}

Bold indicates significant $P$-values.

inbreeding risk increases and genetic variability decreases, thus increasing the fixation of deleterious mutations (Dixo et al., 2009). Given that we expect bold and intermediate individuals to be positively selected in such a context, given their greater tendency to explore novel environments, this may lead to a reduced genetic diversity within fragmented populations, with the rare shy phenotypes being lost. Moreover, previous studies have shown that strong selection on locomotor capacity and mobility may have a significant impact on life-history traits and reproductive output (Phillips et al., 2007; Seebacher and Franklin, 2011). However, to evolve, traits have to be heritable. Previous studies have demonstrated the heritability of morphology and performance (e.g. Le Galliard and Ferrière, 2008; Garland et al., 1990) and exploration behavior has also been found to be heritable in some vertebrate species (Dingemanse et al., 2006; Drent et al., 2003; Pulido et al., 2001). Whether the heritability of these behavioral traits is as strong as that of morphological and performance traits remains, however, unknown. Future studies exploring the genetic basis of these behaviors, as well as the underlying genetic basis of variation in mobility would be especially insightful.

In summary, our data demonstrate the presence of three stable behavioral patterns of exploration in female X. tropicalis. Although males also show three behavioral groups, the behaviors are different between the two sexes, with males being bolder and exploring more than females. Finally, in both males and females, behavior appears to be decoupled from morphology and performance, suggesting that selection can act on both sets of traits independently.

\section{MATERIALS AND METHODS}

Animals

Individuals of $X$. tropicalis were caught in the wild in Cameroon in 2009. An additional 10 individuals that were bred in captivity were added to the data set. Animals were housed at the Muséum National d'Histoire Naturelle (MNHN) in Paris and maintained in 211 tanks mounted on three-shelf stand-alone Xenopus frog racks (Aquaneering, Inc., San Diego, CA, USA) with the water temperature set at $24^{\circ} \mathrm{C}$. This temperature is close to the optimal performance temperature of Xenopus (Herrel and Bonneaud, 2012) and similar to temperatures measured under field conditions for ponds in the forest (Careau et al., 2014). Animals were fed with beef heart and mosquito larvae twice weekly. All individuals were pit-tagged (Nonatec, Rodange, Luxembourg), allowing unique identification of each individual. Data for morphology and performance of these same individuals were published previously (Herrel et al., 2012). Moreover, data on the exploration behavior in males from the same populations have been published recently (Videlier et al., 2014) and are used here for comparison with the data obtained for females. A total of 61 females were used in the current analysis.

\section{Morphology and performance}

Body measures included mass, snout-vent length, ilium length and width, head length, width and height as well as lower jaw length, forelimb segments (humerus, radius, hand and the length of the longest finger) and hindlimb segments (femur, tibia, foot, longest toe length). These measures are important in locomotion and have been described in a previous study (Herrel et al., 2012). Performance measures included swimming velocity and acceleration, as well as terrestrial endurance capacity (time and distance jumped until exhaustion; see Herrel et al., 2012, 2014).

\section{Behavioral analysis}

Frogs were filmed for 60 min with a Quickcam Pro 500 (Logitech, Inc., Romanel-sur-Morges, Switzerland) set at 15 frames $\mathrm{s}^{-1}$. Animals were released into a rectangular tank (height $0.98 \mathrm{~m}$, length $0.40 \mathrm{~m}$, width $0.20 \mathrm{~m}$ ) with a water level of $0.20 \mathrm{~m}$ maintained at $24 \pm 2^{\circ} \mathrm{C}$ (see Videlier et al., 2014) and left quietly for $5 \mathrm{~min}$ before the onset of the recording. Shelters were placed at the two extremities to provide a hiding place. Each individual was tested three times at different times of the day (morning: 09:00 h-12:00 h; early afternoon: 12:00 h-16:00 h; late afternoon: 16:00 h20:00 h) in a randomized way. This allowed us to test the repeatability of behavior across different activity periods. Videos were analyzed using ProAnalyst software (Xcitex, Inc., Cambridge, MA, USA) by tracking all movements of frogs during their exploration of the environment for $1 \mathrm{~h}$ Coordinates of the snout tip were extracted and used to quantify exploration behavior.

The following variables were extracted for each video: the total distance (cm) moved in $1 \mathrm{~h}$; the number of all movements and the number of roundtrips (complete or not); the average, minimal and maximal speed of movement $\left(\mathrm{cm} \mathrm{s}^{-1}\right)$ extracted from the videos; the speed of movement without pauses $\left(\mathrm{cm} \mathrm{s}^{-1}\right)$; the latency to the first, second and last movement (s); the average, minimal and maximal duration of a roundtrip with or without pauses (s); the total duration of exploration with or without pauses (s); the total, average, minimal and maximal time spent hidden between two roundtrips (s); the average, minimal and maximal number of pauses; and the number of roundtrips away from the wall of the aquarium. In total, 29 variables were extracted for each $1 \mathrm{~h}$ video.

All individuals were in good health and were still alive at the time of the submission of this paper and showed no signs of weight loss. Experiments were approved by the institutional ethics committee at the MNHN.

\section{Statistical analyses}

To confirm the assumptions of normality and homoscedascity, all the data were $\log _{10}$ transformed and tested using Shapiro tests. One outlier was detected in the initial exploration of the female data and showed extreme movements in one recording and no movement at all in the two others. This extreme variability was not present in other individuals and thus this female was removed from the data set. The repeatability of each variable was tested using Pearson correlations (Pearson, 1909). Parameters without correlation 
between at least two of the recordings were excluded from the analysis. Thirteen parameters were repeatable: the total distance moved $(\mathrm{cm})$ in $1 \mathrm{~h}$; the number of all movements and roundtrips (complete or not); the maximal speed of movement $\left(\mathrm{cm} \mathrm{s}^{-1}\right)$; the latency of the last movement (s); the minimal and maximal duration of a roundtrip without pauses; the total duration of all movements without pauses; the average, minimal, maximal number of pauses; and the number of roundtrips away from the wall of the aquarium.

To describe variation in the female exploration behavior, a Gaussian mixture model (Fraley and Raftery, 2007) was used without imposed group number. Three groups were detected, and group membership was saved for each individual. To identify the variables that differed between clusters, a MANOVA coupled to subsequent ANOVA and post hoc tests was run on the three clusters. Next, we tested whether behavioral groups differed in performance and morphology using MANOVA. Twelve individuals were excluded because of missing performance data. Finally, we combined previously published data for males ( $N=37$; Videlier et al., 2014) with our data on females to explore differences between sexes in exploration behavior. When combining the two data sets, 15 variables were found to be repeatable across all individuals: the total distance moved in $1 \mathrm{~h}(\mathrm{~cm})$; the number of all movements and complete roundtrips; the maximal, minimal and average speed of movement $\left(\mathrm{cm} \mathrm{s}^{-1}\right)$; the latency of the first, the second and the last movement (s); the average and maximal duration of a roundtrip with pauses (s); the duration of exploration with pauses (s); the average duration spent hidden between two roundtrips (s); the average number of pauses; and the number of roundtrips away from the wall of the aquarium. A Gaussian mixture analysis was run and extracted three groups without a priori group definition. MANOVA and ANOVA were then run to test for differences between sexes and clusters in exploration behavior, and to test for differences in morphology and performance between the groups identified in the overall data set.

All analyses were performed in R (R Development Core Team, 2010) using the Mclust, Class and conventional packages.

\section{Acknowledgements}

We would like to thank L. N. Gonwouo and E. Fokam for their valuable help in the field, and one anonymous reviewer for helpful comments of an earlier version of this paper.

\section{Competing interests}

The authors declare no competing or financial interests.

\section{Author contributions}

C.B. and A.H. conceived the study; M.V. did the experiments; M.V., R.C. and A.H. analyzed the data; all authors drafted and revised the paper.

\section{Funding}

This research was supported by l'Agence Nationale de la Recherche MOBIGEN (ANR-09-PEXT-003 to A.H. and C.B.), a Muséum National d'Histoire Naturelle Action transversale du Muséum (MNHN ATM) grant of the programme 'Biodiversité actuelle et fossile' to A.H., and a Marie Curie reintegration grant to C.B. (FP7PEOPLE-IRG-2008 \#239257).

\section{References}

Berg, M. P., Kiers, E. T., Driessen, G., Van der Heidjen, M., Kooi, B. W., Kuenen, F., Liefting, M., Verhoef, H. A. and Ellers, J. (2010). Adapt or disperse: understanding species persistence in a changing world. Glob. Chang Biol. 16, 587-598.

Brandner, C. (2007). Strategy selection during exploratory behavior: sex differences. Judgement Decis. Mak. 2, 326-332.

Brown, C., Jones, F. and Braithwaite, V. A. (2007). Correlation between boldness and body mass in natural populations of the poeciliid Brachyrhaphis episcopi. J. Fish Biol. 71, 1590-1601.

Careau, V., Biro, P. A., Bonneaud, C., Fokam, E. B. and Herrel, A. (2014). Individual variation in thermal performance curves: swimming burst speed and jumping endurance in wild-caught tropical clawed frogs. Oecologia 175, $471-480$

Carere, C., Drent, P. J., Privitera, L., Koolhaas, J. M. and Groothuis, T. G. G. (2005). Personalities in great tits, Parus major. stability and consistency. Anim. Behav. 70, 795-805.

Carter, A. J., Heinsohn, R., Goldizen, A. W. and Biro, P. A. (2012). Boldness, trappability and sampling bias in wild lizards. Anim. Behav. 83, 1051-1058.
Decker, R. A. and Griffen, B. D. (2012). Correlating context-specific boldness and physiological condition of female sand fiddler crabs (Uca pugilator). J. Ethol. 30 , 403-412.

Desjardins, J. K. and Fernald, R. D. (2009). Fish sex: why so diverse? Curr. Opin. Neurobiol. 19, 648-653.

Dingemanse, N. J. and De Goede, P. (2004). The relation between dominance and exploratory behavior is context-dependent in wild great tits. Behav. Ecol. 15, 1023-1030.

Dingemanse, N. J. and Réale, D. (2005). Natural selection and animal personality. Behaviour 142, 1159-1184.

Dingemanse, N. J., Both, C., Drent, P. J., van Oers, K. and van Noordwijk, A. J. (2006). Repeatability and heritability of exploratory behaviour in great tits from the wild. Anim. Behav. 64, 929-938.

Dingemanse, N. J., Wright, J., Kazem, A. J. N., Thomas, D. K., Hickling, R. and Dawnay, N. (2007). Behavioural syndromes differ predictably between 12 populations of three-spined stickleback. J. Anim. Ecol. 76, 1128-1138.

Dixo, M., Metzger, J. P., Morgante, J. S. and Zamudio, K. R. (2009). Habitat fragmentation reduces genetic diversity and connectivity among toad populations in the Brazilian Atlantic Coastal Forest. Biol. Conserv. 142, 1560-1569.

Drent, P. J., van Oers, K. and van Noordwijk, A. J. (2003). Realized heritability of personalities in the great tit (Parus major). Proc. R. Soc. B Biol. Sci. 270, 45-51.

Dzieweczynski, T. L. and Crovo, J. A. (2011). Shyness and boldness differences across contexts in juvenile three-spined stickleback Gasterosteus aculeatus from an anadromous population. J. Fish Biol. 79, 776-788.

Fraley, C. and Raftery, A. E. (2007). Bayesian regularization for normal mixture estimation and model-based clustering. J. Classif. 24, 155-181.

Garamszegi, L. Z., Eens, M. and Török, J. (2009). Behavioural syndromes and trappability in free-living collared flycatchers, Ficedula albicollis. Anim. Behav. $\mathbf{7 7}$ 803-812.

Garland, T., Bennett, A. F. and Daniels, C. B. (1990). Heritability of locomotor performance and its correlates in a natural population. Experientia 46, 530-533.

Godin, J. G. and Dugatkin, L. A. (1996). Female mating preference for bold males in the guppy, Poecilia reticulata. Proc. Natl. Acad. Sci. USA 93, 10262-10267.

Hedrick, P. W. (2007). Balancing selection. Curr. Biol. 17, R230-R231.

Hedrick, A. V. and Temeles, E. J. (1989). The evolution of sexual dimorphism in animals: hypotheses and tests. Trends Ecol. Evol. 4, 136-138.

Herrel, A. and Bonneaud, C. (2012). Temperature dependence of locomotor performance in the tropical clawed frog, Xenopus tropicalis. J. Exp. Biol. 215 2465-2470.

Herrel, A., Gonwouo, L. N., Fokam, E. B., Ngundu, W. I. and Bonneaud, C. (2012). Intersexual differences in body shape and locomotor performance in the aquatic frog, Xenopus tropicalis. J. Zool. 287, 311-316.

Herrel, A., Vasilopoulou-Kampitsi, M. and Bonneaud, C. (2014). Jumping performance in the highly aquatic frog, Xenopus tropicalis: sex-specific relationships between morphology and performance. PeerJ 2, e661.

Hillers, A., Veith, M. and Rödel, M.-O. (2008). Effects of forest fragmentation and habitat degradation on West African leaf-litter frogs. Conserv. Biol. 22, 762-772.

Husak, J. F., Fox, S. F. and Van Den Bussche, R. (2008). Faster male lizards are better defenders not sneakers. Anim. Behav. 75, 1725-1730.

Kralj-Fišer, S. and Schneider, J. M. (2012). Individual behavioural consistency and plasticity in an urban spider. Anim. Behav. 84, 197-204.

Le Galliard, J. and Ferrière, R. (2008). Evolution of maximal endurance capacity: natural and sexual selection across age classes in a lizard. Evol. Ecol. Res. 10, 157-176.

Mafli, A., Wakamatsu, K. and Roulin, A. (2011). Melanin-based coloration predicts aggressiveness and boldness in captive eastern Hermann's tortoises. Anim. Behav. 81, 859-863.

Monnet, J.-M. and Cherry, M. I. (2002). Sexual size dimorphism in anurans Proc. R. Soc. B Biol. Sci. 269, 2301-2307.

Monroe, M. J. and Alonzo, S. H. (2014). Sexual size dimorphism is not associated with the evolution of parental care in frogs. Ecol. Evol. 4, 4001-4008.

Niemelä, P. T., DiRienzo, N. and Hedrick, A. V. (2012). Predator-induced changes in the boldness of naïve field crickets, Gryllus integer, depends on behavioural type. Anim. Behav. 84, 129-135.

Pearson, K. (1909). Determination of the coefficient of correlation. Sciences 30 , 23-25.

Phillips, B. L., Brown, G. P., Greenlees, M., Webb, J. K. and Shine, R. (2007). Rapid expansion of the cane toad (Bufo marinus) invasion front in tropical Australia. Aust. Ecol. 32, 169-176.

Pulido, F., Berthold, P., Mohr, G. and Querner, U. (2001). Heritability of the timing of autumn migration in a natural bird population. Proc. R. Soc. B Biol. Sci. 268, 953-959. R Development Core Team. (2010). R: A Language and Environment for Statistical Computing. R Foundation for Statistical Computing, Vienna, Austria. Available at: http://www.R-project.org.

Rabb, G. (1969). Fighting frogs. Brookfield Banderlog 37, 4-5.

Rabb, G. and Rabb, M. S. (1965). Effects of isolation onreproductive behavior in the pipid frog Xenopus laevis. Am. Zool. 5, 685. 
Scott, J. P. (1956). The analysis of social organization in animals. Ecol. Soc. Am. 37, 213-221.

Seebacher, F. and Franklin, C. E. (2011). Physiology of invasion: cane toads are constrained by thermal effects on physiological mechanisms that support locomotor performance. J. Exp. Biol. 214, 1437-1444.

Shillito, E. E. (2013). Exploratory behaviour in the short-tailed vole Microtus agrestis. Brill 21, 145-154.

Shine, R. (1979). Sexual selection and sexual dimorphism in the amphibia. Copeia 1979, 297-306.

Sih, A., Bell, A. M., Johnson, J. C. and Ziemba, R. E. (2004). Behavioral Syndromes: an integrative overview. Q. Rev. Biol. 79, 241-277.

Smith, B. R. and Blumstein, D. T. (2008). Fitness consequences of personality: a meta-analysis. Behav. Ecol. 19, 448-455.

Thomson, J. S., Watts, P. C., Pottinger, T. G. and Sneddon, L. U. (2011). Physiological and genetic correlates of boldness: characterising the mechanisms of behavioural variation in rainbow trout, Oncorhynchus mykiss. Horm. Behav. 59, 67-74.

Uher, J., Asendorpf, J. B. and Call, J. (2008). Personality in the behaviour of great apes: temporal stability, cross-situational consistency and coherence in response. Anim. Behav. 75, 99-112. van Oers, K., Drent, P. J., de Goede, P. and van Noordwijk, A. J. (2004). Realized heritability and repeatability of risk-taking behaviour in relation to avian personalities. Proc. R. Soc. B Biol. Sci. 271, 65-73.

Videlier, M., Bonneaud, C., Cornette, R. and Herrel, A. (2014). Exploration syndromes in the frog Xenopus (Silurana) tropicalis: correlations with morphology and performance? J. Zool. 294, 206-213.

Watanabe, N. M., Stahlman, W. D., Blaisdell, A. P., Garlick, D., Fast, C. D. and Blumstein, D. T. (2012). Quantifying personality in the terrestrial hermit crab: different measures, different inferences. Behav. Process. 91, 133-140.

Wells, K. D. (1977). The social behaviour of anuran amphibians. Anim. Behav. 25, 666-693.

Wilson, A. D. M. and Godin, J.-G. J. (2009). Boldness and behavioral syndromes in the bluegill sunfish, Lepomis macrochirus. Behav. Ecol. 20, 231-237.

Wittern, A. K. and Berggren, A. (2007). Natal dispersal in the North Island Robin (Petroica longipes): the importance of connectivity in fragmented habitats dispersion. Avian Conserv. Ecol. 2, 2.

Wolf, M., van Doorn, G. S., Leimar, O. and Weissing, F. J. (2007). Life-history trade-offs favour the evolution of animal personalities. Nature 447, 581-584.

Woolbright, L. L. (1983). Sexual selection and size dimorphism in anuran amphibia. Am. Nat. 121, 110-119. 